\title{
Non-Immersive Virtual Reality for Upper Limb Rehabilitation in Stroke Survivors - A Feasibility Study
}

Grigoras A.V., Matei D., Ignat E.B.

Corresponding author: Grigoras A.V., E-mail: valer.grigoras@gmail.com

\begin{abstract}
We present the results of a feasibility study for the use of a commercially available non-immersive virtual reality system (SeeMe ${ }^{\circledR}$ ) in upper limb motor rehabilitation of stroke survivors. The study included 8 chronic phase stroke survivors. All patients received 12 sessions with the system over 2 weeks, each session comprising 20 minutes of motor training using a sequence of 8 serious games. Outcome measures included the Fugl-Meyer assessment scale - upper limb section (FMA), the Stroke Impact Scale (SIS), the ingame assessment procedure of the SeeMe system and a feedback questionnaire.

Following the rehabilitation program, we found no change on the clinical scales. Although some of the system generated parameters (score, endurance and reaction time or movement amplitude for each game) have improved significantly, in 3 of the 8 games none of the recorded variables has shown significant changes, with only one game showing significant improvement in 2 out of 3 parameters. The satisfaction questionnaire did not generally correlate with game performance (although this has happened in some of the games), but reflected correctly the increased interest of the subjects for the intervention and also their awareness of its real influence on motor abilities.

This initial pilot study indicates that the SeeMe virtual reality system has the potential to be of use in clinical settings as a complement to conventional therapy. Future studies should include larger number of subjects, longer training duration, use more sensitive/dedicated measurements of improvement, and focus on a single game/exercise type.
\end{abstract}

Key words: non-immersive virtual reality, stroke, rehabilitation, upper limb

\section{Introduction.}

Control of the motor activities (in both real life and experimental situations) is shared between semiautomatic systems and voluntary, attention dependent and resources consuming networks [1,2]. In addition to direct damage of the motor control structures, stroke leads to a diminished computation capability of the brain. Natural, non-demanding activities (as are learned motor gestures and behaviors) become then dependent on direct allocation of the main attention flow. Stroke survivors are not able to cope with multiple stimuli and are not able to perform multiple tasks at once. Various studies have highlighted the differences between "indoors" performance and "real life situations" [3,4], emphasizing the need for personalized and adapted interventions in terms of objectives and means to attain them.

Traditional rehabilitation aims at improving motor performance. May it be concentrating on elementary movement or muscle tone control or on functional gestures, it is essentially perceived as a therapeutic gesture, a "drug” or "specialized intervention". As motor learning is dependent on personal emotional participation and involvement [5], this perception may be of help when performed in a standardized setting. However, due to its nature, the "white coat motivating effect” lacks in home independent rehabilitation. Since home continuous exercise is a key element of the processes that might be required for the continuous adaptations, sustaining long time patient involvement is a delicate matter [6]. According to Kleim and Jones, some of the principles that, on a global scale, influence neuroplasticity are salience, specificity, transference, intensity, repetition, timing [7]. In plain English, in order for an exercise to translate into a useful behavior it needs to be similar to that act, to be interesting, and to be subject to numerous repetitions at the right moment.

Another important issue is the mismatch between the needs and expectations (of both doctors and patients) and available therapeutic interventions. In this perspective, most (if not all) of the rehabilitation approaches have but limited impact on the quality of life and social integration of significantly disabled stroke patients. In most cases, recovery is not a matter of reparation but of compensation - all rehabilitation uses available structures and functions to compensate and to make better use of remaining resources.

The direct consequence of this is the continuous quest for better methods. Since we cannot directly address the responsible neural circuits, we hope that a more cunning manipulation of the whole might increase the impact and durability of the intervention. Virtual reality is one of the means used to increase the impact 
of simple motor training. In short, the brain were tricked to associate valuable subjective traits to the basic training, thus increasing its valor (salience) and chances that it affects the existing state of equilibrium [8]. This is done by creating a virtual environment where the patient moves and behaves purposefully "use of interactive simulations created with computer hardware and software" allows users with" opportunities to be engaged in environments that appear and feel similar to real-world objects and events" [9]. More systems that are complex use 3D goggles or complex projection systems to immerse the patient into the virtual reality, eventually adding haptic stimulation or treadmills/moving platforms that address gait and balance. However, the approach remains mostly reserved to specialized facilities, and consequently is available for limited amounts of time for a limited number of patients. In an attempt to cover the needs for affordable home rehabilitation, gaming class motion sensors and systems have been adapted to "serious games" - e.g., Nintendo Wii ${ }^{\circledR}$ (Nintendo) [10], Playstation EyeToy ${ }^{\circledR}$ (Sony) [11,12], Xbox 360/XboxOne ® (Microsoft) [13]. Although the sensation they provide is less realistic (as the avatar of the user is usually placed inside the virtual environment - system put the user inside the computer instead of projecting the generated environment around him), these systems do provide some of the features that constitute the theoretical basis of the approach [14].

In this study we have evaluated the possible benefices of a short rehabilitation program using a Kinect $\mathbb{B}$ based virtual rehabilitation system - the See Me ${ }^{\circledR}$ system of Brontes Processing - in chronic stroke patients.

\section{Materials and method}

Between Mars and July 2016, 8 stroke survivors had been select from the Neurology Clinic of Iaşi Rehabilitation Hospital to be part of our study. The ethics committee of the "Grigore T. Popa" Medical University has approved the research protocol. Subjects agreed to take part in the study and signed the informed consent.

The inclusion criteria were 6-12 months after an acute CT confirmed stroke, ability to maintain autonomous seating or standing position, moderate motor deficit (Fugl Meyer scale - upper limb scores between 15 and 50). Significant comorbidity, presence of a neglect syndrome, major apraxic, perceptual or cognitive deficits (MMSE less than 25), aphasia, or other neurological, neuromuscular, orthopedic conditions or visual disturbances, as well as history of multiple strokes were the exclusion criteria.

Average age of the patients was $46.75 \pm 16.56$ years. Time since stroke was $9.75 \pm 1.75$ months (with a minimum stroke age 6 months and a maximum stroke age of 12 months). Stroke was ischemic in $75 \%$ of the patients (in the territory of the middle cerebral artery) and hemorrhagic (frontal lobe hematoma) in the rest. Education was college grade or above for $50 \%$ of the patients, high school level for $37.5 \%$ and only 1 subject had completed the secondary school level.

Motor abilities were evaluate before and after the rehabilitation program. We have used the Fugl Meyer assessment score (upper limb components) (FMA) [15] and the Stroke Impact Scale (SIS) [16]. Initial FMA average score was $39.5 \pm 16.36$ (with 5 of the patients in the range of 50 and 2 patients with a score of 19). Average SIS was $15.13 \pm 6.66$, with values ranging from 7 to 22 .

The VR rehabilitation program consisted in 12 daily sessions of training with the See Me system. The seated subject performs the tasks while watching his movements on the display where he interacts with virtual objects. Depending on the particular games, the subject may see himself as an avatar facing frontward or as mirror image, which faces him with the virtual space beneath them. The therapist can to modify game parameters according to each patient`s motor performance.

Each patient went through a succession of 8 games: Warm up, Gym, React, Space, Cleaner, Ball, Maze, Oarsman and Sorter (fig. 1). In "Warm up" the patient touches 9 numbered boxes; in "Cleaner" he cleans a window; in "Space" he has to shoot enemy space crafts; in "React" he has to touch as quickly as possible the balls appearing on his sides; in "Ball" he has to kick or duck different objects approaching him; in "Maze" he has to push a cube through a maze in order to reach the target; in "Oarsman" he has to paddle in a canoe down a river; in "Gym" he has to kick different objects according to a color code using his arms and feet and in "Sorter" he has to bimanually guide falling balls using a stick. The succession of games (with $30 \mathrm{~s}$ pauses between them) complete a 20 minutes session (Warm up - 90s, Cleaner - 90s, 
Space - 120s, React - 120s, Gym- 120s, Ball - 120s, Maze - 120s, Oarsman - 90s, Sorter: 90s).

Data from each training session was record for each of the games - reaction time, endurance, precision and range of motion (ROM).

All of the 8 subjects underwent standard rehabilitation therapy as well as the VR therapy.

At the end of the training program all subjects have completed a questionnaire (based on the SFQ (Short Feedback Questionnaire)[17] which was conceived to measure user experience when using new technologies, as is VR), assessing perception and satisfaction at patient/user level. Patients have evaluated the overall experience, realism, connection, improvement of their in game performance, satisfaction generated by game achievements, efficiency of avatar control, ease of adaptation to game requirements, movement of the paretic arm, self-evaluation of paretic limb participation in daily activities (dressing, eating, washing) and overall usefulness of VR training as part of motor rehabilitation. Evaluation was done on 4 steps scales (values from 0 to 3 , with 3 coding for the most useful/a lot/very and 0 for disliked/not at all/worse/not useful).

Due to the low number of subjects, statistical analysis was limited; descriptive parameters (average, standard deviation), have been calculated with the Excel package (Microsoft). For the ANOVA analysis, Student's $T$ test and Pearson's $p$ correlation coefficient we have used the SPSS v.18 (IBM Analytics) statistical package.

\section{Results:}

None of the primary efficacy indicators (FMA or SIS) has changed following the 12 days VR training program in any of the patients. Table 1 shows initial (as well as final) values of the two main scores and some of the clinical parameters.

\begin{tabular}{|l|l|l|l|l|l|l|l|l|}
\hline Patient & RC & LV & VC & TP & AV & CV & VB & LI \\
\hline $\begin{array}{l}\text { FMA } \\
\text { initial/final }\end{array}$ & $19 / 19$ & $50 / 50$ & $50 / 50$ & $48 / 48$ & $19 / 19$ & $50 / 50$ & $30 / 30$ & $50 / 50$ \\
\hline $\begin{array}{l}\text { SIS } \\
\text { initial/final }\end{array}$ & $8 / 8$ & $20 / 20$ & $22 / 22$ & $14 / 14$ & $8 / 8$ & $20 / 20$ & $7 / 7$ & $22 / 22$ \\
\hline
\end{tabular}

Table 1. Initial and final FMA upper limb and SIS values
Overall, all of the system generate parameters have improved, but few have reached statistical significance. ANOVA analysis of the score changes in the 10 separate games did not point out any significant differences, as did not the ANOVA analysis of the endurance changes. In Table 2 can be seen the average changes of those parameters (presented as \% from the initial values) in all the games. Score and endurance were been calculated for all games, while amplitude of movements with the impaired upper limb and reaction time are produced for some of them.

\begin{tabular}{|c|c|c|c|c|c|c|c|c|}
\hline & $\begin{array}{l}\text { Clea } \\
\text { ner }\end{array}$ & Ball & $\begin{array}{l}\text { Reac } \\
\mathrm{t}\end{array}$ & $\begin{array}{l}\text { Sort } \\
\text { er }\end{array}$ & $\begin{array}{l}\text { Oars } \\
\text { man }\end{array}$ & $\begin{array}{l}\text { Gy } \\
\mathrm{m}\end{array}$ & $\begin{array}{l}\text { Maz } \\
\mathrm{e}\end{array}$ & $\begin{array}{l}\text { Spa } \\
\text { ce }\end{array}$ \\
\hline Score & $\begin{array}{l}220 . \\
49 \pm \\
258 . \\
3\end{array}$ & $\begin{array}{l}223 . \\
74 \pm \\
233 . \\
93 *\end{array}$ & $\begin{array}{l}184 . \\
87 \pm \\
375 . \\
33\end{array}$ & $\begin{array}{l}65.7 \\
9 \pm \\
68.6 \\
4^{*}\end{array}$ & $\begin{array}{l}12.5 \pm \\
51.28\end{array}$ & $\begin{array}{l}65.6 \\
7 \pm \\
136 . \\
1\end{array}$ & $\begin{array}{l}51.8 \\
9 \pm \\
79.8 \\
4\end{array}$ & $\begin{array}{l}63 . \\
6 \pm \\
82 . \\
47\end{array}$ \\
\hline Endurance & $\begin{array}{l}161 . \\
8 \pm \\
248 . \\
2\end{array}$ & $\begin{array}{l}25.4 \\
\pm \\
21.3 \\
3^{*}\end{array}$ & $\begin{array}{l}165 . \\
62 \pm \\
217 . \\
14^{*}\end{array}$ & $\begin{array}{l}105 . \\
9 \pm \\
280 . \\
8\end{array}$ & $\begin{array}{l}22.3 \pm \\
27.4^{*}\end{array}$ & $\begin{array}{l}79.7 \\
2 \pm \\
175 . \\
9\end{array}$ & $\begin{array}{l}12.5 \\
\pm \\
36.3 \\
4\end{array}$ & $\begin{array}{l}3.4 \\
4 \pm \\
15 . \\
93\end{array}$ \\
\hline Amplitude & $\begin{array}{l}239 . \\
17 \pm \\
636 . \\
29\end{array}$ & $\begin{array}{l}27.3 \\
\pm \\
46.7 \\
74\end{array}$ & - & $\begin{array}{l}41.9 \\
9 \pm \\
49.3 \\
3\end{array}$ & $\begin{array}{l}-1.7 \pm \\
11.6\end{array}$ & - & - & $\begin{array}{l}5.7 \\
9 \pm \\
24 . \\
26\end{array}$ \\
\hline $\begin{array}{l}\text { Reaction } \\
\text { Time }\end{array}$ & - & - & $\begin{array}{l}9.51 \\
\pm \\
64.7\end{array}$ & - & - & $\begin{array}{l}- \\
49.1 \\
\pm \\
22.8 \\
*\end{array}$ & - & - \\
\hline
\end{tabular}

Table 2. Average change in computer generated parameters after 12 training sessions (\% from initial values). Stars have been added where the parameter variation (before and after training) reaches statistical significance

In "Cleaner" the score was higher but did not reach statistical significance $(p=0.06)$. For "Ball" both the score $(p=0.02)$ and endurance $(p=0.01)$ have significantly increased. In "React" endurance has significantly increased $(\mathrm{p}=0.02)$ while the score has shown only a non-significant increase $(p=0.09)$. The score increased significantly in "Sorter" $(p=0.03)$, while in "Oarsman" only endurance was significantly higher $(p=0.03)$. In "Gym" reaction time was significantly lower $(\mathrm{p}=0.04)$ with neither score nor endurance changing significantly. There is a significant difference in reaction time evolution in the 2 tasks that calculate it $(\mathrm{p}=0.034)$ - on average, while in "react" reaction time increases, in "Gym" it decreases.

We have tried to establish correlations between clinical scores and different system scores. In "Ball" 
SIS and FMA have significantly correlated with the final movement amplitude $(\mathrm{r}=0.945, \mathrm{p}<0.001$ and $r=0.732, p=0.039$ respectively), but not with the amplitude change ( $p=0.13$ and 0.153 ). In "Oarsman" both SIS and FMA have correlated with the increase of amplitude in affected upper limb movement $(\mathrm{r}=0.908$ and $\mathrm{r}=0.866$ respectively, $\mathrm{p}=0.002$ and 0.005 respectively).

The satisfaction questionnaire average response value (per test and subject) was 2.05 (2 meaning "good"). The average rating for each question (with lower values representing worse appreciation) is present in figure 1.

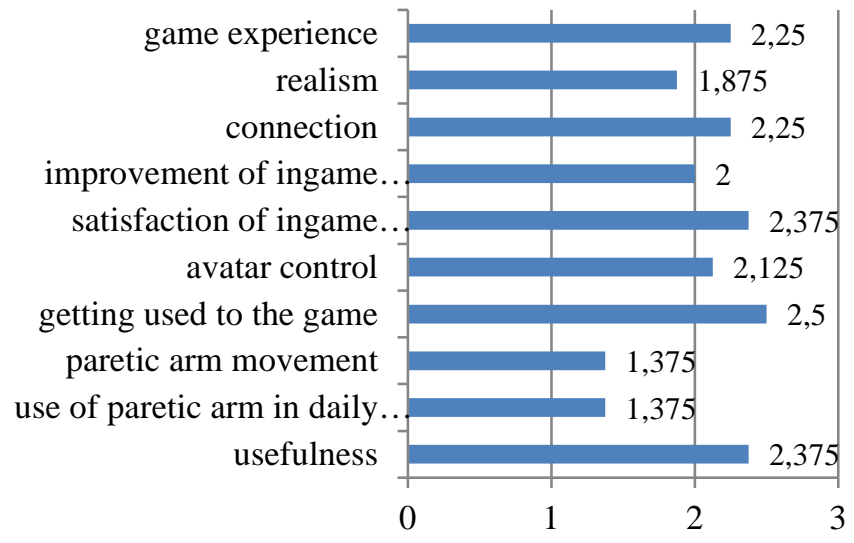

Fig.1. Averaged responses for each question of the satisfaction questionnaire

The average rating/question for each subject ranged from 1.3 to 2.5 , with a tendency to positively correlate with education level $(r=0.677, p=0.065)$. Objective scores and history parameters did not correlate with averaged questionnaire scores. Correlation analysis for the score changes and individual responses at the questionnaire questions has shown only little significance - the only game in which improvement actually correlated with the response to the "improvement of the in game performance" question was "Ball" $(r=0.765, p=0.027)$. None of the other score changes correlated with game experience, realism, connection, avatar control, game performance improvement, getting used to the game or usefulness scores. However, usefulness has correlated with game performance improvement $(\mathrm{r}=0.73, \mathrm{p}=0.04)$, realism correlated with game connection $(\mathrm{r}=0.709, \mathrm{p}=0.049)$ and with satisfaction of in game performance $(r=0.712, p=0.048)$. Strangely, FMA had a strong inverse correlation with game experience $(\mathrm{r}=-0.881, \mathrm{p}=0.004)$. Endurance variation at the "Space" game has correlated with paretic arm movement question responses ( $\mathrm{r}=0,734$, $\mathrm{p}=0.034$ ); endurance variation for "Oarsman" and "React" have correlated with in game performance improvement $(\mathrm{r}=0.797, \mathrm{p}=0.018$ and $\mathrm{r}=0.798$, $\mathrm{p}=0.018$ respectively). Amplitude of movement change in "Oarsman" has negatively correlated with game experience $(r=-0.73, p=0.04)$.

\section{Discussion}

A number of studies have reported various degrees of improvement after training with VR devices, either using commercially available games (e.g., Sports ${ }^{\circledR}$ and Sports $2 \circledR$ for Xbox 360® [18]) or custom made applications, running on hybrid platforms (computer and motion sensor). (for a review see [19]). However, reviews and meta-analyses usually point out that, despite the theoretical potential, studies in this field do not manage to prove a clear cut advantage over classical rehabilitation and that further research is needed [19, 20, 21, 22] (although some authors find sufficient proof to state this $[23,24]$, at least in some aspects of the rehabilitation).

The "See Me" system homepage advertises it as a tool to "practice and evaluate your patient's strength, endurance, range of motion, postural control, reaction time, proprioception, quality of movement, perception, divided attention and memory" [25]. Previous research with the system include mostly small low quality studies regarding its usefulness for the evaluation and treatment of spatial neglect [26], assessment of executive functions [27], walking and balance [28], evaluation of balance and fear of falling [29], balance improvement [30].

The system generates a non-immersive VR. The subject sees his avatar interacting with virtual objects and mimicking his movements. Although this may bring some advantages, as action observation could contribute to learning [31], it requires the imposition of an added sensorimotor transformation [32]. It is different from a first person experience where the virtual environment replaces the visual field of the subject and he interacts directly with the virtual environment - in [33] authors find significant differences in scores in a comparison between a less and a more immersive version of the same game.

Most of the subjects (7 out of 8) have performed the exercises in standing position. While this allows involvement of the lower limbs, seated position avoids fatigue due to supplementary effort and limits 
the possibility to replace movement of the paretic arm with moving/leaning the whole body towards that side. Seated position also avoids the presence of the investigator in the immediate vicinity of the patient, reported as a potential bias in standing/walking studies due to the inability of the camera to differentiate between the two persons [18]. We have not evaluated a possible impact on balance and lower limb function, as we have concentrated on upper limb function alone. Due to the characteristics of the sensor and movement algorithms, superimposed body parts are sometimes difficult to separate. We consider that in our study this has reduced the capacity of the system to ignore compensatory movements.

The games provide a wide array of movement, and the possibility to personalize the difficulty (in the current study all the participants have used the easiest level in all games). In “Cleaner”, global positioning movements of the upper limb are required (on all axes of movement, favoring extension), as the subject uses his hand to wipe clean as many mirrors as possible, (mirrors appear in front of him). There is no requirement for a specific strategy, but different areas/positions of the limb can be favored by choosing a specific location of the mirror. In "React" stationary targets appear and the subject is required to touch them, favoring reaching movements of the upper limb. In "Ball” targets are moving, so that coordination, reaching movements and reacting rapidly to moving objects appearance (including anticipating the trajectory and point of contact) are necessary. "Maze" requires the patient to guide an object - more precise and coordinated movements are necessary. In "Sorter”, bilateral arm movement leans a bat that directs falling balls. Bilateral arm movement (paddling) is required in "Oarsman" (mostly anterior-posterior, without much height variation), while in "Space" arms are shot out stretched in order to reach extreme positions. "Gym" requires quick punching movements in different directions.

Choosing the right game can have profound consequences on the results. We found significant changes of scores in just two of the 8 games ("Ball" and "Sorter") and reaction time decreased with statistical significance in one ("Gym”). Endurance has increased in three games, but this can be related (more than the overall scores) to increased knowledge of the game. Coincidence of both parameters increasing significantly has appeared just for "Ball".
Overall, multiple (but essentially similar) types of upper limb movement are represented in the featured games, with a clear focus on large positioning movements. Since the tracking ability of the sensor for movement of the hand and fingers is limited, prehension and release are not approaches. While practice of global extension patterns could improve release and finger extension, we do not expect this type of movements to change as a direct result of the training program. Since 24 of the 66 possible points on the Fugl-Meyer scale originate from wrist and hand movements [15] and the same situation arises with SIS, where only three of the 8 sections are directly related to upper limb function [16], one could consider these scores as not sensitive enough for our rehabilitation program. However, none of the sub scores has improved in any of the two tests. This could be trace to multiple factors and that would need to be change in the event of a larger study.

Intensity of training is important. While more action is reported to take place on virtual reality rehabilitation than in conventional therapy in general [34], authors reported an overall median of $570 \mathrm{~min}$ of VR (or computer games) therapy delivered, with duration ranging from 20 to 60 minutes per session, and 8 to 36 sessions [35]. Our study has totaled 240 minutes of training over 12 sessions (mainly correlated to the usual length of stay in our service). Although the software allows it, we have not recorded the number of repetitions of the functional gestures. Increasing duration and intensity may contribute a better impact on functional parameters.

In direct relation to the intensity of training, stroke age is important in terms of receptivity. Duration since stroke was around 10 months in our study. Although reports place the greater part of recovery within the first 3 months [36], in a large VR study [37], effect size on FMA was similar in patients with strokes 3-12 and >12 months old, and smaller (although significantly more important than classical therapy) in patients at less than 3 months from the cerebrovascular event. Later stages recovery is more dependent on neuroplasticity and learning [38], and hence on the general principles that govern it. We found no significant correlation of stroke age with any of the parameter changes recorded after the training program.

One of the putative advantages of serious games is the availability of alternate measures of performance. In [39] a consensus panel states "recovery trials need to 
consider serially applied kinematic/kinetic measurements alongside clinical assessments to distinguish between restitution and compensation". Leaving aside the fact that movement amplitude is the only data we have collected that might be consider "kinematic", our experiment does not support the use of game scores and parameters as surrogate markers for motor function. We found limited correlations between the established measures (FMA and SIS) and the scores, endurance, reaction time. From this angle, movement amplitude correlates the most with the two scores. A potential bias comes from the limitation of the maximum amplitude to 180 degrees (due to ingame evaluation algorithm).

The subjective perception of the VR/serious game was realistic. Subjects rated favorably the interaction, and were less enthusiastic about effects on arm mobility or use in current activities. Improving their game performance was clearly not mistaken to be an improvement of the motor functions. Responses have shown a good correlation with the game performance. More severely disabled patients have appreciated the game than patients in a better physical/neurological shape (FMA inversely correlated with game experience).

According to the questionnaire, interaction with the system was been appreciated favorably. Improving one's performance was rewarding and motivating to continue. Although we did not apply a standardized measure for depression and self-appreciation, the subjects that improved their performance have also reported improvements in their self-image.

Also not captured by the study measures, compensatory movements have been present in more severely impaired patients (mostly RC and AV, but also VB). Moving the paretic limb with the healthy one or entirely relying on it to fulfill the game tasks were frequent behaviors and required repeated intervention from the investigator in order to be suppressed. It is probable that without the constant reminding that they should try to exercise the affected limb some of the patients would have entirely avoided use of the paretic side. In the idea of an independent home use this might be catastrophic as it would only enhance learned non-use. Some degree of supervision might then be necessary for a suited use of the system. In terms of costs, the $4500 \mathrm{E}$ price is prohibitive for home use. Since, for example, Kinect ${ }^{\circledR}$ and commercially available games (with costs that do not exceed 300E) have shown efficacy in stroke rehabilitation [18], the advanced features of the SeeMe would be useless in independent home use. Customization of the games to fit to the neurological impairment as well as an optimal use of all parameters that SeeMe provide, require the intervention of trained therapists.

The small sample size is the most important limitation of our study. To evaluate the impact of this rehabilitation approach more data that are robust are necessary. Among other, our patient sample is relatively homogenous in regard of the time since stroke but in terms of initial severity of the neurological deficit it is clearly parted in a more disabled (FMA $<20)$ and a less disabled $(\mathrm{FMA} \approx 50)$ group, with subject numbers too reduced to allow separate analysis.

Game ability has improved, but we cannot state with certainty whether this signals a functional improvement below clinical scores' sensitivity or whether this is simply caused by learning of the game requirements. A classical therapy reference group with proportionally sized intensity of exercise is necessary to evaluate the efficacy of the SeeMe system. However, allowing a 3 sessions period for the subjects to "get accustomed" with the games before measurement begins (setting the reference point at the $4^{\text {th }}$ session rather than at the $1^{\text {st }}$ ) might eliminate the "skill" factor. Although in some of the games there was a tendency for better progress in the beginning, the data was not enough to draw a clear conclusion on this issue.

\section{Conclusion}

This pilot study aims to establish the most appropriate clinical and technical setting that would maximize the benefit of SeeMe training for rehabilitation. Since our intention was not to prove that the method is valuable, but more to find the proper way to do that, we consider that we have reached our objectives.

Training with the SeeMe system for 12 days, 20 $\mathrm{min} /$ day did not lead to any functional impact. The main cause is probably insufficient intensity of training. In our opinion, using a large palette of games is not likely to be beneficial unless more time is dedicated to each type of functional exercise. It is possible that if we used a single game (since the better correlations appeared with "Ball" this would be our choice for a more focused study) a narrower but stronger improvement would be perceivable on 
clinical scales. Use of more restricted components of the FMA or SIS may also contribute to sensitize the results and adding measures for global disability and stroke severity (National Institute of Health stroke scale; modified Rankin Scale) would be useful to compare with other studies [39]. Training session duration should be at least double (or two sessions should be performed) for at least 20 days.

Subject feedback was favorable in terms of satisfaction and easiness of use, and the questionnaire we have used is appropriate to capture the important data. However, to evaluate impact on depression and quality of life a further study should include tools as Beck depression inventory and EQ5D.

The SeeMe system is easy to use and configure and might facilitate motor function improvement, but in our opinion, it would be better use in a controlled setting and not as home based rehabilitation. Further studies are necessary to validate its clinical effect in motor rehabilitation of stroke patients beyond the research possibilities it provides.

\section{Abbreviations}

FMA: Fugl-Meyer scale, upper limb components

SIS: Stroke Impact Scale

VR: Virtual Reality

MMSE: Mini Mental State Examination

\section{Acknowledgements}

We thank Dr. V. Bohotin for reviewing the manuscript.

\section{Funding}

The SeeMe software was provided free of charge for testing purposes by BRONTES PROCESSING Sp. z o.o. (Ltd.) (Poland)

\section{Conflict of interests: none to declare Authors' contributions}

GAV conceived the study, conducted the experiment and collected the data. IEB led the data analysis and drafted the manuscript. MD contributed the data analysis, contributed to and critically appraised the manuscript and the revision. All authors read and approved the final manuscript.

\section{Bibliography}

1. Hikosaka O. Neural systems for control of voluntary action - a hypothesis. Adv Biophys. 1998;35:81-102.

2. de Wit S, Barker RA, Dickinson AD, Cools R. Habitual versus goal-directed action control in Parkinson disease. Journal of Cognitive Neuroscience. 2011;23(5):1218-1229. DOI:10.1162/jocn.2010.21514

3. Lord SE, McPherson K, McNaughton HK, Rochester L, Weatherall M. Community ambulation after stroke: how important and obtainable is it and what measures appear predictive? Arch Phys Med Rehabil. 2004;85:234-239.

4. Maclellan MJ, Richards CL, Fung J, Mcfadyen BJ. Use of segmental coordination analysis of nonparetic and paretic limbs during obstacle clearance in community-dwelling persons after stroke Phys. Med. Rehabil. 2013;5:381-391.

5. Hodges NJ, Lohse KR, Wilson A, Lim SB, Mulligan D. Exploring the dynamic nature of contextual interference: previous experience affects current practice but not learning. J Mot Behav. 2014;46(6):455-67.

6. Kaur G, English C, Hillier S. Physiotherapists systematically overestimate the amount of time stroke survivors spend engaged in active therapy rehabilitation: an observational study. J Physiother. 2013;59(1):45-51.

7. Kleim JA, Jones TA. Principles of experience-dependent neural plasticity: implications for rehabilitation after brain damage. Journal of Speech, Language, and Hearing Research. 2008;51:S225-S239. doi:10.1044/10924388(2008/018).

8. Levin MF, Weiss PL, Keshner EA. Emergence of virtual reality as a tool for upper limb rehabilitation: incorporation of motor control and motor learning principles. Phys Ther. 2015;95(3):415-425. doi: 10.2522/ptj.20130579

9. Weiss P, Kizony R, Feintuch U, Katz N. Virtual reality in neurorehabilitation. In: Selzer M, Cohen L, Gage F, Clarke S, Duncan P, eds. In: Textbook of Neural Repair and Rehabilitation. Cambridge, UK: Cambridge University Press; 2006:182-197.

10. Saposnik G, Teasell R, Mamdani M, Hall J, McIlroy W, Cheung D, Thorpe KE, Cohen LG, Bayley M. Stroke Outcome Research Canada (SORCan) Working Group. Effectiveness of virtual reality using Wii gaming technology in stroke rehabilitation: a pilot randomized clinical trial and proof of principle. Stroke. 2010;41(7):1477-84. doi: 10.1161/STROKEAHA.110.584979.

11. Mouawad MR, Doust CG, Max MD, McNulty PA.Wii-based movement therapy to promote improved upper extremity function post-stroke: a pilot study. J Rehabil Med. 2011 May;43(6):527-33. doi: 10.2340/16501977-0816.

12. Neil A, Ens S, Pelletier R, Jarus T, Rand D. Sony PlayStation EyeToy elicits higher levels of movement than the Nintendo Wii: implications for stroke rehabilitation. Eur J Phys Rehabil Med 2013;49:13-21.

13. Asskın A, Atar E, Koçyiğit H, Tosun A. Effects of Kinectbased virtual reality game training on upper extremity motor recovery in chronic stroke. Somatosens Mot Res. 2018 Mar;35(1):25-32. doi: 10.1080/08990220.2018.1444599.

14. Saposnik G, Cohen LG, Mamdani M, Pooyania S, Ploughman M, Cheung D, Shaw J, Hall J, Nord P, Dukelow $\mathrm{S}$, et al. Efficacy and safety of nonimmersive virtual reality 
exercising in stroke rehabilitation (EVREST): a randomised, multicentre, single-blind, controlled trial. Lancet Neurol. 2016; 15(10):1019-27.

15. Fugl-Meyer AR, Jaasko L, Leyman I, Olsson S, Steglind S. The post-stroke hemiplegic patient. A method for evaluation of physical performance. Scand J Rehabil Med. 1975;7:1331.

16. Duncan PW, Bode RK, Min Lai S, Perera S. Rasch analysis of a new stroke specific outcome scale: the Stroke Impact Scale. Arch Phys Med Rehabil. 2003;84:950-963.

17. Kizony R, Katz N, Rand D, Weiss PL. A Short Feedback Questionnaire (SFQ) to enhance client-centered participation in virtual environments. Proceedings of 11th annual cybertherapy conference: Virtual healing: Designing reality (2006)

18. Park DS, Lee DG, Lee K, Lee GK, Effects of Virtual Reality Training using Xbox Kinect on Motor Function in Stroke Survivors: A Preliminary Study. Journal of Stroke and Cerebrovascular Diseases. 2017; 26(10):2313-2319.

19. Howard M. A meta-analysis and systematic literature review of virtual reality rehabilitation programs. Computers in Human Behavior: 2017;70:317-327. https://doi.org/10.1016/j.chb.2017.01.013.

20. Lucca LF. Virtual Reality And Motor Rehabilitation Of The Upper Limb After Stroke: A Generation Of Progress? J Rehabil Med 2009; 41:1003-1006.

21. Rose T, Nam CS, Chen K. Immersion of virtual reality for rehabilitation - Review. Applied Ergonomics. 2018; 69:153-161.

22. Vinas-Diz S, Sobrido-Prieto M. Virtual reality for therapeutic purposes in stroke: A systematic review. Neurología. 2016;31(4):255—277.

23. Corbetta D, Imeri F, Gatti R. Rehabilitation that incorporates virtual reality is more effective than standard rehabilitation for improving walking speed, balance and mobility after stroke: a systematic review. Journal of Physiotherapy. 2015;61:117-124.

24. Gauthier LV, Richter TA, George LC, Schubauer KM. Gaming for the Brain: Video Gaming to Rehabilitate the Upper Extremity After Stroke. In Neuromodulation, Second Edition. 2018 Elsevier. http://dx.doi.org/10.1016/B978-012-805353-9.00033-4

25. http://www.seeme.rehab/Clinicians.html

26. Sugarman H, Burstin A, Weisle-Eichler A, Brown R. Use of Novel Virtual Reality System for the Assessment and Treatment of Unilateral Spatial Neglect: A Feasibility Study. Paper presented at the International Conference on Virtual Rehabilitation, Zurich 2011.

27. Nir-Hadad SY, Weiss PL, Waizman A, Schwartz N, Kizony R. A virtual shopping task for the assessment of executive functions: Validity for people with stroke, Neuropsychological Rehabilitation. 2015;27:5:808-833. DOI: 10.1080/09602011.2015.1109523

28. Dunsky A, Fishbein P, Hutzle Y. Dual-task training using virtual reality: Influence on walking and balance in three post stroke survivors. International Journal of Therapies and Rehabilitation Research, 2013, 2(2): 22-34. DOI: 10.5455/ijtrr.00000021

29. Laufer Y, Burstin A, Brown R, Korsensky O, Hutzler Y. Rapid Stepping Time on 'Virtual' Objects Correlates with
Measures of Balance and Fear of Falling in Elderly Individuals. Physiotherapy. 2015; 101(Supplement 1):e189.

30. Hadash R, Amrani S, Pinsker A, Lotan M, Weiss T. Improving Balance of Individuals with Intellectual and Developmental Disability through a Virtual Reality Intervention Program. Palaestra. 2015;29(4):19-19.

31. McGregor HR, Gribble PL. Changes in visual and sensorymotor resting-state functional connectivity support motor learning by observing. J. Neurophysiol. 2015;114(1):677688. http://dx.doi. org/10.1152/jn.00286.2015.

32. Messier J, Kalaska JF. Differential effect of task conditions on errors of direction and extent of reaching movements. Exp Brain Res. 1997;115:469-478.

33. Hondori HM, Khademi M, Dodakian L, McKenzie A, Lopes $\mathrm{CV}$, Cramer SC. Choice of Human-Computer Interaction Mode in Stroke Rehabilitation. Neurorehabilitation and neural repair. 2016;30(3):258-265. doi:10.1177/1545968315593805

34. Brunner I, Skouen JS, Hofstad H, et al. Is upper limb virtual reality training more intensive than conventional training for patients in the subacute phase after stroke? An analysis of treatment intensity and content. BMC Neurology. 2016;16:219. doi:10.1186/s12883-016-0740-y.

35. Lohse KR, Hilderman CG, Cheung KL, Tatla S, Van der Loos HF. Virtual reality therapy for adults post-stroke: a systematic review and meta-analysis exploring virtual environments and commercial games in therapy. PLoS One 2014; 9:e93318

36. Wade DT, Langton-Hewer R, Wood VA, Skilbeck CE, Ismail HM. The hemiplegic arm after stroke: measurement and recovery. J. Neurol. Neurosurg. Psychiatry. 1983;46:521-524. 10.1136/jnnp.46.6.521

37. Turolla A, Dam M, Ventura L, et al. Virtual reality for the rehabilitation of the upper limb motor function after stroke: a prospective controlled trial. Journal of NeuroEngineering and Rehabilitation. 2013;10:85. doi:10.1186/1743-0003-1085.

38. Kwakkel G, Kollen B, Lindeman E. Understanding the pattern of functional recovery after stroke: facts and theories. Restor Neurol Neurosci 2004;22(3-5):281-99.

39. Kwakkel G, Lannin NA, Borschmann K, English C, Ali M, Churilov L, Saposnik G, Winstein C, van Wegen EE, Wolf SL, Krakauer JW, Bernhardt J. Standardized measurement of sensorimotor recovery in stroke trials: Consensus-based core recommendations from the Stroke Recovery and Rehabilitation Roundtable. Int J Stroke. 2017 Jul;12(5):451461. doi: 10.1177/1747493017711813. 